\title{
Papers to appear in forthcoming issues
}

Adler, R. L., Nowicki, T., Świrszcz, G. and Tresser, C.

Akin, E., Glasner, E., Huang, W., Shao, S. and Ye, X.

Antonevich, A. B., Bakhtin, V. I. and Lebedev, A. V.

Arthur Robinson, E. and Şahin, A. A.

Ashwin, P. and Goetz, A.

Badea, C., Grivaux, S. and Müller, V.

Bakhtin, V. I.

Barbot, T. and Maquera, C.

Barge, M., Diamond, B., Hunton, J. and Sadun, L.

Barral, J. and Bhouri, I.

Barreira, L. and Gelfert, K.

Bezuglyi, S., Kwiatkowski, J., Medynets, K. and

Solomyak, B.

Bowen, L.

Bowen, L.

Brown, A. W.

Calegari, D. and Fujiwara, K.

Chavaudret, C.

Chazottes, J.-R., Gambaudo, J.-M. and Ugalde $\mathrm{E}$.

Chu Q.

Comman, H. and Rivera-Letelier, J.

Cornea, O., de Rezende, K. A. and da Silveira, M. R.

Cornulier, Y. and Tessera, R.
Convex dynamics with constant input

Sufficient conditions under which a transitive system is chaotic

$T$-entropy and variational principle for the spectral radius of transfer and weighted shift operators

Rank-one $\mathbb{Z}^{d}$ actions and directional entropy

Cone exchange transformations and boundedness of orbits

Epsilon-hypercyclic operators

On $t$-entropy and variational principle for the spectral radius of weighted shift operators

Transitivity of codimension one Anosov actions of $\mathbb{R}^{k}$ on closed manifolds

Cohomology of substitution tiling spaces

Multifractal analysis for projections of Gibbs and related measures

Dimension estimates in smooth dynamics

Invariant measures on stationary Bratteli diagrams

Entropy for expansive algebraic actions of residually finite groups

Non-abelian free group actions: Markov processes, the Abramov-Rohlin formula and Yuzvinskii's formula

Constraints on dynamics preserving certain hyperbolic sets

Combable functions, quasimorphisms, and the central limit theorem

Reducibility of quasiperiodic cocycles in linear Lie groups

Zero-temperature limit of one-dimensional Gibbs states via renormalization: the case of locally constant potentials

Multiple recurrence for two commuting transformations

Large deviation principles for non-uniformly hyperbolic rational maps

Spectral sequences in Conley's theory

A characterization of relative Kazhdan property $\mathrm{T}$ for semidirect products with abelian groups 
Coronel, A. D.

Dal'bo, F., Peigné, M., Picaud, J.-C. and Sambusetti, A.

Danilenko, A. I. and Ryzhikov, V. V.

de la Llave, R. and Windsor, A.

Demers, M. F.

Ding, F., Pan, J., Wang, S. and Yao, J.

Downarowicz, T. and Lacroix, Y.

Durieu, O. and Volný, D.

Eroğlu, K. I., Rohde, S. and Solomyak, B.

Eynard, $\mathrm{H}$.

Falconer, K. and Samuel, T.

Fletcher, A. N. and Nicks, D. A.

Frantzikinakis, N., Johnson, M., Lesigne, E. and Wierdl, M.

Fujino, M.

Gaidashev, D. and Koch, H.

Galatolo, S. and Pacifico, M. J.

Gavish, M.

Gentile, G.

Glasner, E., Lemańczyk, M. and Weiss, B.

Guilloux, A.

Gutman, Y.

Harvey, N. and Peres, Y.

Hochman, M.

Hong, S.

Host, B. and Kra, B.

Jenkinson, O. and Steel, J.

Jordan, T. and Rams, M.

Kamae, T.
Cohomological equation on dynamical systems arising from Delone sets

On the growth of quotients of Kleinian groups

Mixing constructions with infinite invariant measure and spectral multiplicities

Livšic theorems for non-commutative groups including diffeomorphism groups and results on the existence of conformal structures for Anosov systems

Functional norms for Young towers

Only rational homology spheres admit $\Omega(f)$ to be union of DE attractors

The law of series

On sums of indicator functions in dynamical systems

Quasisymmetric conjugacy between quadratic dynamics and iterated function systems

A connectedness result for commuting diffeomorphisms of the interval

Dixmier traces and coarse multifractal analysis

Quasiregular dynamics on the $n$-sphere

Powers of sequences and convergence of ergodic averages

$C^{*}$-algebras arising from substitutions

Period doubling in area-preserving maps: an associated one-dimensional problem

Lorenz like flows: exponential decay of correlations for the Poincaré map, logarithm law, quantitative recurrence

Measures with uniform scaling scenery

Quasi-periodic motions in strongly dissipative forced systems

A topological lens for a measure-preserving system

A brief remark on orbits of $\operatorname{SL}(2, \mathbb{Z})$ in the euclidean plane

Embedding $\mathbb{Z}^{k}$-actions in cubical shifts and $\mathbb{Z}^{k}$-symbolic extensions

An invariant of finitary codes with finite expected square root coding length

Non-expansive directions for $\mathbb{Z}^{2}$ actions

The zeta functions of renewal systems

Nil-Bohr sets of integers

Majorization of invariant measures for orientation-reversing maps

Multifractal analysis of weak Gibbs measures for non-uniformly expanding $C^{1}$ maps

Mixing properties of numeration systems coming from weighted substitutions 
Kennedy, J., Raines, B. E. and Stockman, D. R.

Kočan, Z., Kornecká-Kurková, V. and Málek, $M$.

Koch, H. and Kocić, S.

Kwiatkowska, A. and Solecki, S.

Larsen, N. S.

Leplaideur, R.

Leplaideur, R., Oliveira, K. and Rios, I.

Levin, G.

Levine, L.

Li, S. and Shen, W.

Llibre, J. and Valls, C.

Lohrmann, P.

Los, J.

Lozano-Rojo, Á.

Łuczak, A.

Markarian, R., Pujals, E. and Sambarino, $\mathrm{M}$.

Matsumoto, K.

Mihailescu, E.

Miles, R.

Neishtadt, A. and Treschev, D.

Nogueira, A.

Olivier, E.

Osipenko, G.

Patrão, M.

Pavlov, R.

Pelayo, A. and Tolman, S.

Pikuła, R.

Pivato, M. and Yassawi, R.

Qiao, J., Yin, Y. and Gao, J.
Basins of measures on inverse limit spaces for the induced homeomorphism

Entropy, horseshoes and homoclinic trajectories on trees, graphs and dendrites

A renormalization group approach to quasiperiodic motion with Brjuno frequencies

Spatial models of Boolean actions and groups of isometries

Crossed products by abelian semigroups via transfer operators

Thermodynamic formalism for a family of non-uniformly hyperbolic horseshoes and the unstable Jacobian

Equilibrium states for partially hyperbolic horseshoes

Multipliers of periodic orbits in spaces of rational maps

Parallel chip-firing on the complete graph: Devil's staircase and Poincaré rotation number

An improved real $C^{k}$ Koebe principle

Analytic integrability of quadratic-linear polynomial differential systems

Normalisation holomorphe de structures de Poisson

Infinite sequence of fixed point free pseudo-Anosov homeomorphisms

An example of a non-uniquely ergodic lamination

The eigenvectors of semigroups of positive maps on von Neumann algebras

Pinball billiards with dominated splitting

A class of simple $C^{*}$-algebras arising from certain nonsofic subshifts

Asymptotic distributions of preimages for endomorphisms

Finitely represented closed-orbit subdynamics for commuting automorphisms

Polymorphisms and adiabatic chaos

Lattice orbit distribution on $\mathbb{R}^{2}$

Uniqueness of the measure with full dimension on sofic affine-invariant subsets of the 2-torus

Symbolic image and invariant measures of dynamical systems

Entropy and its variational principle for noncompact metric spaces

Perturbations of multidimensional shifts of finite type

Fixed points of symplectic periodic flows

Enveloping semigroups of unipotent affine transformations of the torus

Embedding Bratteli-Vershik systems in cellular automata

Feigenbaum Julia sets of singularities of free energy 
Raja, C. R. E.

Rebelo, J. C.

Rechtman, A.

Rees, M.

Robinson, Jr, E. A. and Şahin, A. A.

Roesch, P.

Skalski, A.

Sumi, H.

Thompson, D.

Timár, Á.

Turaev, D.

Worm, D. T. H. and Hille, S. C.

$\mathrm{Xu}, \mathrm{J}$. and Jiang, S.

Xue, Y.-M. and Kamae, T.

Yokoyama, T.

Zhao, X.
On the existence of ergodic automorphisms in ergodic $\mathbb{Z}^{d}$-actions on compact groups

On transverse rigidity for singular foliations in $\left(\mathbb{C}^{2}, 0\right)$

Existence of periodic orbits for geodesible vector fields on closed 3-manifolds

Multiple equivalent matings with the aeroplane polynomial

Rank-one $\mathbb{Z}^{d}$ actions and directional entropy

Cubic polynomials with a parabolic point

On automorphisms of $C^{*}$-algebras whose Voiculescu entropy is genuinely non-commutative

Dynamics of postcritically bounded polynomial semigroups III: classification of semi-hyperbolic semigroups and random Julia sets which are Jordan curves but not quasicircles

A thermodynamic definition of topological pressure for non-compact sets

Invariant colorings of random planar maps

Diffeomorphisms which cannot be topologically conjugate to diffeomorphisms of a higher smoothness

Ergodic decompositions associated with regular Markov operators on Polish spaces

Reducibility for a class of nonlinear quasi-periodic differential equations with degenerate equilibrium point under small perturbation

Partitions by congruent sets and optimal positions

Fixed points of certain Anosov maps on Riemannian manifolds

Non-singular Smale flows on three-dimensional manifolds and Whitehead torsion 


\section{CAMBRIDGE}

\section{Noteworthy Titles from Cambridge!}

\section{NIST Handbook of Mathematical Functions}

Edited by Frank W. J. Olver, Daniel W. Lozier, Ronald F. Boisvert, and Charles W. Clark

Modern developments in theoretical and applied science depend on knowledge of the properties of mathematical functions, from elementary trigonometric functions to the multitude of special functions. These functions appear whenever natural phenomena are studied, engineering problems are formulated, and numerical simulations are performed. They also crop up in statistics, financial models, and economic analysis. Using them effectively requires practitioners to have ready access to a reliable collection of their properties.

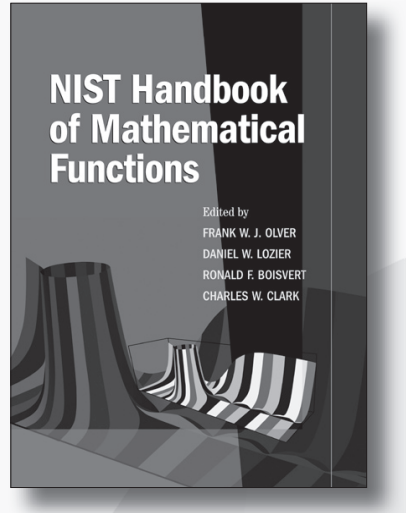

This handbook results from a 10-year project conducted by the National Institute of Standards and Technology with an international group of expert authors and validators. It is destined to replace its predecessor, the classic but long-outdated NBS Handbook of Mathematical Functions, edited by Abramowitz and Stegun.

\$99.00: Hardback: 978-0-521-19225-5: 966 pp.

\$50.00: Paperback: 978-0-521-14063-8

\section{Conformal Fractals}

Ergodic Theory Methods

Feliks Przytycki and Mariusz URbański

London Mathematical Society Lecture Note Series

\$78.00: Paperback: 978-0-521-43800-1: 375 pp.

\section{Geometric Analysis of Hyperbolic Differential Equations:} An Introduction

\section{S. AlinHAC}

London Mathematical Society Lecture Note Series

\$50.00: Paperback: 978-0-521-12822-3: 135 pp.

\section{Multidimensional Stochastic Processes as Rough Paths}

\section{Theory and Applications}

Peter K. Friz and Nicolas B. Victolr

Cambridge Studies in Advanced Mathematics

\$85.00: Hardback: 978-0-521-87607-0: 670 pp.

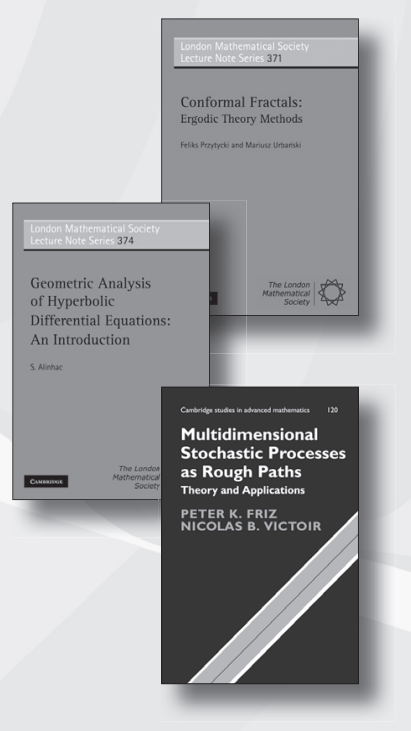

Prices subject to change. 


\section{CAMBRIDGE JOURNALS}

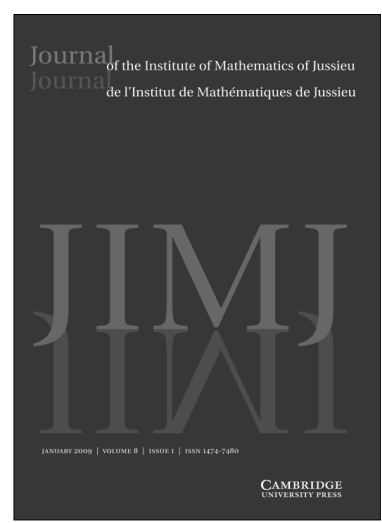

Journal of the Institute of Mathematics of Jussieu is available online at http://journals.cambridge.org/jmj

To subscribe contact Customer Services

in Cambridge:

Phone +44 (0)1223 326070

Fax $+44(0) 1223325150$

Email journals@cambridge.org

in New York:

Phone +1 (845) 3537500

$\mathrm{Fax}+1$ (845) 3534141

Email

subscriptions_newyork@cambridge.org

\section{Journal of the Institute of} Mathematics of Jussieu

Published for the Institute of Mathematics of Jussieu

\section{Editor-in-Chief}

Michael Harris, Institut de Mathematiques de Jussieu, France

The Journal of the Institute of Mathematics of Jussieu covers all domains in pure mathematics. It includes important research articles from areas such as operator algebra, number theory, algebraic and Lie groups, differential and symplectic geometry, partial differential equations, Banach spaces, potential theory, mathematical physics, and probability. The internataional editorial board naturally draws upon the broad expertise of the highly prestigious Jussieu Mathematical Institute in Paris.

\section{Price information is available at http://journals.cambridge.org/jmj}

\section{Free email alerts}

Keep up-to-date with new material - sign up at http://journals.cambridge.org/alerts 


\section{INSTRUCTIONS FOR CONTRIBUTORS}

\section{Editorial Policy}

The journal welcomes high quality contributions on topics closely related to dynamical systems and ergodic theory. Submissions in the field of differential geometry, number theory, operator algebra, differential, topological, symbolic, measurable dynamics and celestial and statistical mechanics are especially welcome. Expository survey papers and reviews of relevant books will be published from time to time.

\section{Submission of typescripts}

Two copies of the manuscript should be submitted to an executive or managing editor whose interest is closest to the material of their article. In case of doubt authors may send manuscripts to the Managing Editors at the University of Warwick. A paper may also be submitted electronically in postscript form as an attachment to an email, i.e. not in the body of an email. Please also send the $\mathrm{T}_{\mathrm{E}} \mathrm{X}$ file. If you are unable to do this, please submit the manuscript in printed form. The editor in charge of the paper will acknowledge receipt of the paper. It is important that authors inform the editor of any changes of postal and/or e.mail address while their paper is under consideration.

The editor will send the manuscript to the referee electronically, if possible, and also airmail a copy to him/her. However, the editor can decide if the material is inappropriate for the journal and will also make the final decision regarding acceptance or rejection. Should you need further information you should communicate in the first instance with the editor in charge of your paper. In an attempt to decrease the journal's backlog an editor may decline a submission (without using the normal refereeing process) if he/she considers that a 'reasonable' referee might not recommend the paper sufficiently strongly, or (possibly) if the paper is more than 30 pages long. Such summary action, with its inherent risk of unfairness, seems preferable to introducing a moratorium (the only other alternative) and has the advantage that delayed negative decisions might be avoided. This policy will be reviewed at a later date.

Submission of a paper is taken to imply that it has not been previously published and that it is not being considered for publication elsewhere. Authors of articles published in the journal assign copyright to Cambridge University Press (with certain rights reserved) and you will receive a copyright assignment form for signature on acceptance of your paper.

The journal strongly recommends submission of accepted papers in LATEX using the ETDS LATEX class file. Papers that use this class file will be processed more efficiently. A $\mathrm{LAT}_{\mathrm{E} X 2 \mathrm{e}}$ file etds.cls is available via anonymous ftp from the Cambridge University Press site at ftp.cup.cam.ac.uk in the directory /pub/texarchive/journals/latex/etds-cls/. In case of difficulties with these files, please contact etds@ sunrise-setting.co.uk or the Journal editorial office at etds@maths.warwick.ac.uk. Alternatively, authors may use "article" style.

On final acceptance of a paper, authors should send the LTEX source code on disc including the figures (line figures only) and all author-defined macro and style files, to the Managing Editors, together with a hard copy produced using the same file. Discs should be in Apple Mac or PC format and will not be returned. The publisher reserves the right to typeset any article by conventional means if the author's $\mathrm{T} \mathrm{X}$ code presents problems in production.

\section{Typescript}

Papers should be typed, double-spaced, on one side only and with generous margins. The pages must be numbered.

The first page should give the title, the author's name and institution, and a short abstract intelligible to mathematicians.

The title, while brief, must be informative (e.g. A new proof of the ergodic theorem, whereas Some applications of a theorem of Birkhoff would be useless).

\section{Notation}

It is important that mathematician's expressions are clear to a printer (who is not a mathematician). For instance, $n_{k}(n$ sub $k$ ) is common usage, but avoid if possible using $c \operatorname{sub} n \operatorname{sub} k$. Fractions are generally best expressed by a solidus. Complicated exponents like $\exp \left\{z^{2} \sin \theta /\left(1+y^{2}\right)\right\}$ should be shown in this and no other way.

In the typescript, italics, small capitals and capitals are specified by single, double and triple underlining. Bold-faced type is shown by wavy underlining.

It helps if displayed equations or statements which will be quoted later are numbered in order on the right of their line. They can then be referred to by, for example, 'from (7)'.

The author must enable the printer (if necessary by pencilled notes in the margin) to distinguish between similar symbols such as $o, O$, o, $\mathrm{O}, 0 ; x, \mathrm{X}, \times ; \phi, \Phi, \varnothing ; 1,1 ; \varepsilon, \epsilon ; \kappa, k$.

There is no need to underline Greek or script letters provided these are clearly typed. Any special symbols should be explained on a separate sheet of directions for the printer.

If an author wishes to mark the end of the proof of a theorem, the sign $\square$ may be used

Footnotes should be avoided.

\section{Diagrams}

Figures and drawings should be on separate sheets in black ink or produced by computer to comparable quality. Photocopies are acceptable only if they are as clear as the originals. Symbols, legends and captions should be given on a transparent overlay. Each text figure must be numbered as Figure 1, Figure 2, .. and its intended position clearly indicated in the typescript. The author's name in pencil must be on all separate sheets of diagrams. Figures should be used sparingly and only when they greatly clarify the exposition.

Tables

Tables should be numbered (above the table) and set out on separate sheets. Indicate the position of each in the text as for figures.

\section{References}

References should be collected at the end of the paper numbered in alphabetical order of the author's names. A reference to a book should give the title, in italics, and then in roman type the publisher's name and the place and year of publication:

[4] N. Dunford and J. T. Schwartz. Linear Operators. Part I. Wiley, New York, 1958.

A reference to a paper should give in italics the title of the periodical, the number of the volume and year, and the beginning and end pages of the paper. Titles should be abbreviated as in Mathematical Reviews:

[6] J. E. Littlewood. The 'pits effect' for functions in the unit circle. J. Analyse Math. 23 (1970), 236-268.

\section{Proofs}

Authors receive one set of proofs for correction. If excessive alterations to the original manuscript are requested after the paper has been typeset, the author will be charged the cost of resetting. For papers with more than one author the proofs are sent to the first named author unless the editor receives other instructions. It is important that proofs are corrected and returned promptly.

\section{Offprints}

No paper offprints are provided, but the corresponding author will be sent the pdf of the published article. Print offprints may be purchased at extra cost at proof stage.

This journal issue has been printed on FSC-certified paper and cover board. FSC is an independent, non-governmental, not-for-profit organization established to promote the responsible management of the world's forests. Please see www.fsc.org for information. 


\title{
Ergodic theory and dynamical systems
}

\author{
VOLUME 30 PART 3 JUNE 2010
}

\section{CONTENTS}

Bressaud, X., Durand, F. and Maass, A. On the eigenvalues of finite rank Bratteli-Vershik dynamical systems

Bressaud, X., Hubert, P. and Maass, A. Persistence of wandering intervals in self-similar affine interval exchange transformations

Bruin, H., Demers, M. and Melbourne, I. Existence and convergence properties of physical measures for certain dynamical systems with holes

Froyland, G., Lloyd, S. and Quas, A. Coherent structures and isolated spectrum for Perron-Frobenius cocycles

Gupta, C. Extreme-value distributions for some classes of non-uniformly partially hyperbolic dynamical systems

Hayashi, S. An extension of the ergodic closing lemma

Hochman, M. On the automorphism groups of multidimensional shifts of finite type

Leibman, A. Multiple polynomial correlation sequences and nilsequences

Levin, G. and Światek, G. Measure of the Julia set of the Feigenbaum map with infinite criticality

Nayak, T. and Prasad, M. G. P. Iteration of certain meromorphic functions with unbounded singular values

San Martin, L. A. B. and Seco, L. Morse and Lyapunov spectra and dynamics on flag bundles

Sun, P. Zero-entropy invariant measures for skew product diffeomorphisms

Yarmola, T. Degenerate random perturbations of Anosov diffeomorphisms

Papers to appear in forthcoming issues 